\title{
Synthesis of a Series of Novel Tetra-tert-butylcalix[4]arene Linked to 1,2,4-Triazole and 1,3,4-Oxadiazole Derivatives
}

\section{Zahra Dono Ghezelbash and Karim Akbari Dilmaghani*}

\author{
Department of Organic Chemistry, Faculty of Chemistry, Urmia University, Urmia, 57159, Iran \\ * Corresponding author: E-mail: k.adilmaghani@urmia.ac.ir \\ Tel: (+98)914-443-1392; Fax: (+98)44-357153-165
}

Received: 29-05-2016

\begin{abstract}
A series of tetra-tert-butylcalix[4] arene linked to 1,2,4-triazole-5-thiones and 1,3,4-oxadiazole-5-thiones derivatives at lower rim were synthesized by the reaction of 1,2,4-triazole-5-thione and 1,3,4-oxadiazole-5-thione with 5,11,17,23-tetra-tert-butyl-25,27-bis(3-bromopropoxy)-26,28-dihydroxycalix[4] arene (2). The synthesized compounds were characterized by FT-IR, ${ }^{1} \mathrm{H}$ NMR, ${ }^{13} \mathrm{C}$ NMR spectral data, elemental analysis and ESI-MS.
\end{abstract}

Keywords: Tetra-tert-butylcalix[4]arene, 1,2,4-triazole-5-thione, 1,3,4-oxadiazole-5-thione, macrocycle

\section{Introduction}

During the last three decades, due to the rapid development of drug resistance, numerous searches for new bioactive compounds have been conducted through screening and synthesizing new compounds with chemical characteristics clearly different from those of existing agents. Calixarene derivatives not only have been used in macromolecular chemistry, catalysis and separation techniques but they also have attracted much interest for their potential use in drug design and delivery. ${ }^{1-7}$ Their controlled functionalization has allowed the use of these compounds in supramolecular chemistry as scaffolds for the construction of various receptors, carriers, and spatial organizers. Calix[4]arenes have been investigated for their biological proprieties; for example hydrophilic derivatives have shown interesting levels of activity against bacteria, cancerous cell, fungi, enveloped virus thrombosis, and fibrosis diseases. ${ }^{8-14}$

1,2,4-Triazole-5-thiones and 1,3,4-oxadiazole-5thiones scaffolds are heterocyclic compounds containing five membered rings and their derivatives have been studied due to their diverse pharmacological proprieties, such as antibacterial and antifungal, ${ }^{15-22}$ antimicrobial, ${ }^{23-25}$ anti-inflammatory, ${ }^{22,26}$ and antiviral activities. ${ }^{27,28}$ Calix[4] arenes represent a third generation of supramolecular hosts after cyclodextrins and crown ethers, with unique three-dimensional structures. Tetra-tertbutylcalix[4] arene (1) was prepared by Gutsche ${ }^{29}$ method as white crystals in good yield through a one-step condensation of $p$-tert-butyl phenol with formaldehyde and sodium hydroxide in diphenyl ether under reflux condition. Calix[4]arenes can be selectively functionalized at either narrow rim if the derivatization of calix[4]arenes occurs at the phenolic oxygen or wide rim if it occurs at the para position after the removal of para-tert-butyl groups. Recently, a review paper on the pharmaceutical uses of calixarene derivatives has been published. ${ }^{30}$

In our previous works, ${ }^{31-33}$ we reported the synthesis and antibacterial properties with new series of thioglycoside heterocyclic derivatives of 1,2,4-triazole-5-thiones and 1,3,4-oxadiazole-5-thiones. Here we report the synthesis of novel tetra-tert-butylcalix[4]arene derivatives containing a series of 1,2,4-triazole-5-thione and 1,3,4oxadiazole-5-thione moieties at the lower rim; we expected that antibacterial properties of these compounds $\mathbf{4 a}-\mathbf{e}$ and $\mathbf{6} \mathbf{f}-\mathbf{k}$ will be of interest.

\section{Result and Discussion}

1-Substituted-4-phenylthiosemicarbazides 2a-e were prepared by the condensation of carboxylic acid hydrazides 1a-k with phenylisothiocyanate under reflux in ab- 
solute ethanol. The cyclodehydration of 1-substituted-4phenylthiosemicarbazides $\mathbf{2 a - e}$ were carried out in alkaline medium followed by acidification with dilute hydrochloric acid to give 1,2,4-triazole-5-thiones 3a-e according to the literature ${ }^{34-36}$ (Scheme 1).

The main synthetic route for substituted 1,3,4-oxadiazole-5-thiones $\mathbf{5 f}-\mathbf{k}$ involves an initial reaction between carboxylic acid hydrazides $\mathbf{1 a}-\mathbf{k}$ and carbon disulfide in basic ethanol solution, followed by acidification with dilute hydrochloric acid which results in the precipitation of oxadiazole. These compounds were synthesized according to the literature method ${ }^{37,38}$ (Scheme 1).

The synthesis of 5,11,17,23-tetra-tert-butyl-25,27bis(3-bromopropoxy)-26,28-dihydroxycalix[4]arene (2) was carried out by the reaction of tetra-tert-butylcalix[4]arene (1) with 1,3-dibromo propane and $\mathrm{K}_{2} \mathrm{CO}_{3}$ in acetonitrile according to the procedure reported in the literature $^{39}$ (Scheme 2).

The existence of thiol-thione tautomerism is known for the compounds $\mathbf{3 a}-\mathbf{e}$ and $\mathbf{5 f}-\mathbf{k}$ and generally one forms is predominant. ${ }^{40-43}$ Replacement of the bromine of compound 2 with 1,2,4-triazole-5-thiones $\mathbf{3 a}-\mathbf{e}$ or 1,3,4oxadiazole-5-thiones $\mathbf{5 f}-\mathbf{k}$ was carried out in aqueous alkaline media in dimethylformamide to afford the corres- ponding coupled calix[4]arenes, i.e. compounds $\mathbf{4 a - e}$ and 6f-k, respectively (Scheme 3 ).

An important property of calixarenes is that the aromatic rings can rotate and produce different conformations (cone, partial cone, 1,3-alternate and 1,2-alternate). The cone conformation is the most favored among the four conformations due to the very strong intramolecular hydrogen bonding between four $\mathrm{OH}$ groups at the lower rim of the unsubstituted calix[4]arene. The conformation of a specific calix[4]arene sample can be easily distinguished by its characteristic ${ }^{1} \mathrm{H}-\mathrm{NMR}$ patterns arising from the $\mathrm{ArCH}_{2} \mathrm{Ar}$ methylene protons. ${ }^{44-47}$ Cone conformation is a preferred conformation which corresponds to the minimum energy. The cone conformer is identified by one characteristic pair of doublets of the Ar- $\mathrm{CH}_{2}-\mathrm{Ar}$ methylene protons (AB system, a coupling constant ${ }^{2} J=12-15 \mathrm{~Hz}$ is characteristic for geminal protons). The ${ }^{1} \mathrm{H}-\mathrm{NMR}$ spectra indicating that $\mathbf{4 a}-\mathbf{e}$ and 6f $-\mathbf{k}$ exist in the cone conformation. Different factors such as the size of the functional groups attached, interactions between the groups, and temperature, among others, influence the rings to either freely rotate or become fixed in a conformation. We suppose that two factors might influence for this conformation selectivity. Firstly,

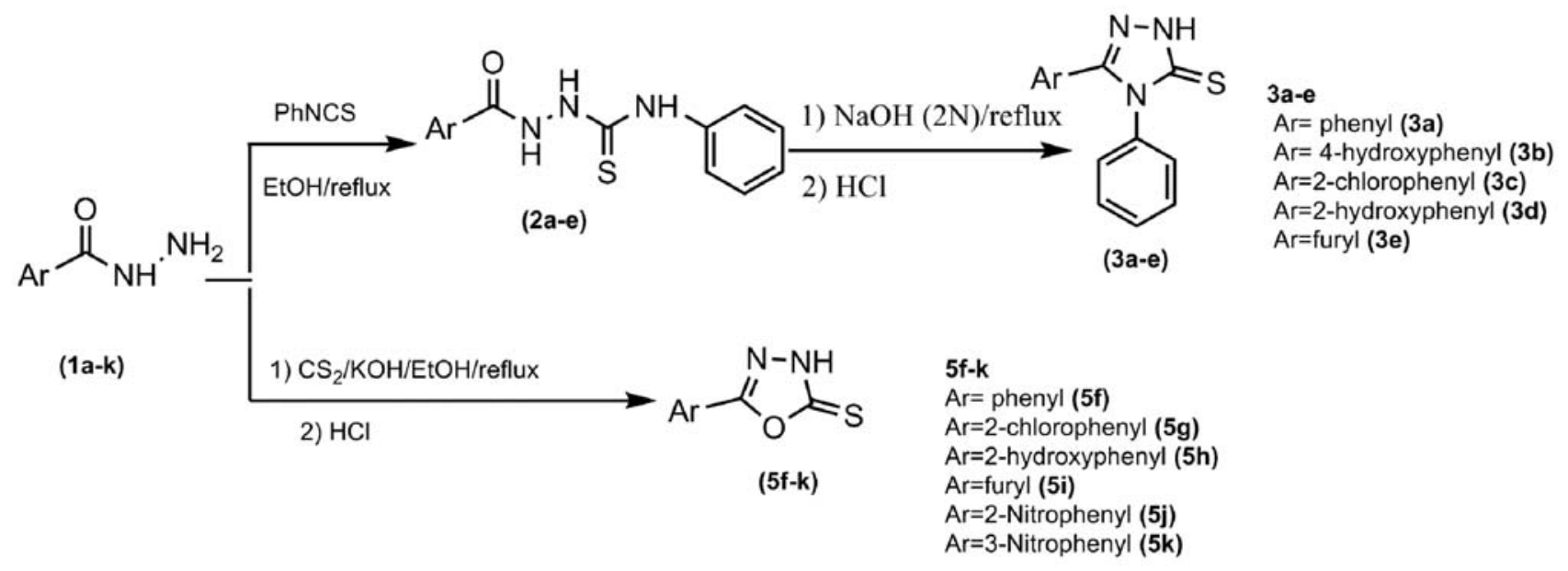

Scheme 1. Synthesis of 1,2,4-triazole-5-thione and 1,3,4-oxadiazole-5-thione derivatives.

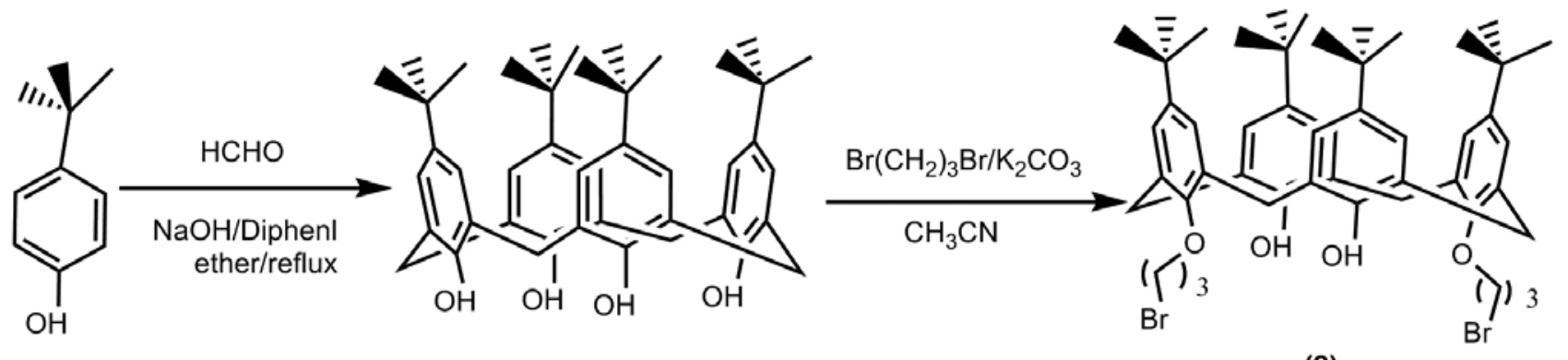

(1)

(2)

Scheme 2. Synthesis of 5,11,17,23-tetra-tert-butyl-25,27-bis(3-bromopropoxy)-26,28-dihydroxycalix[4]arene. 


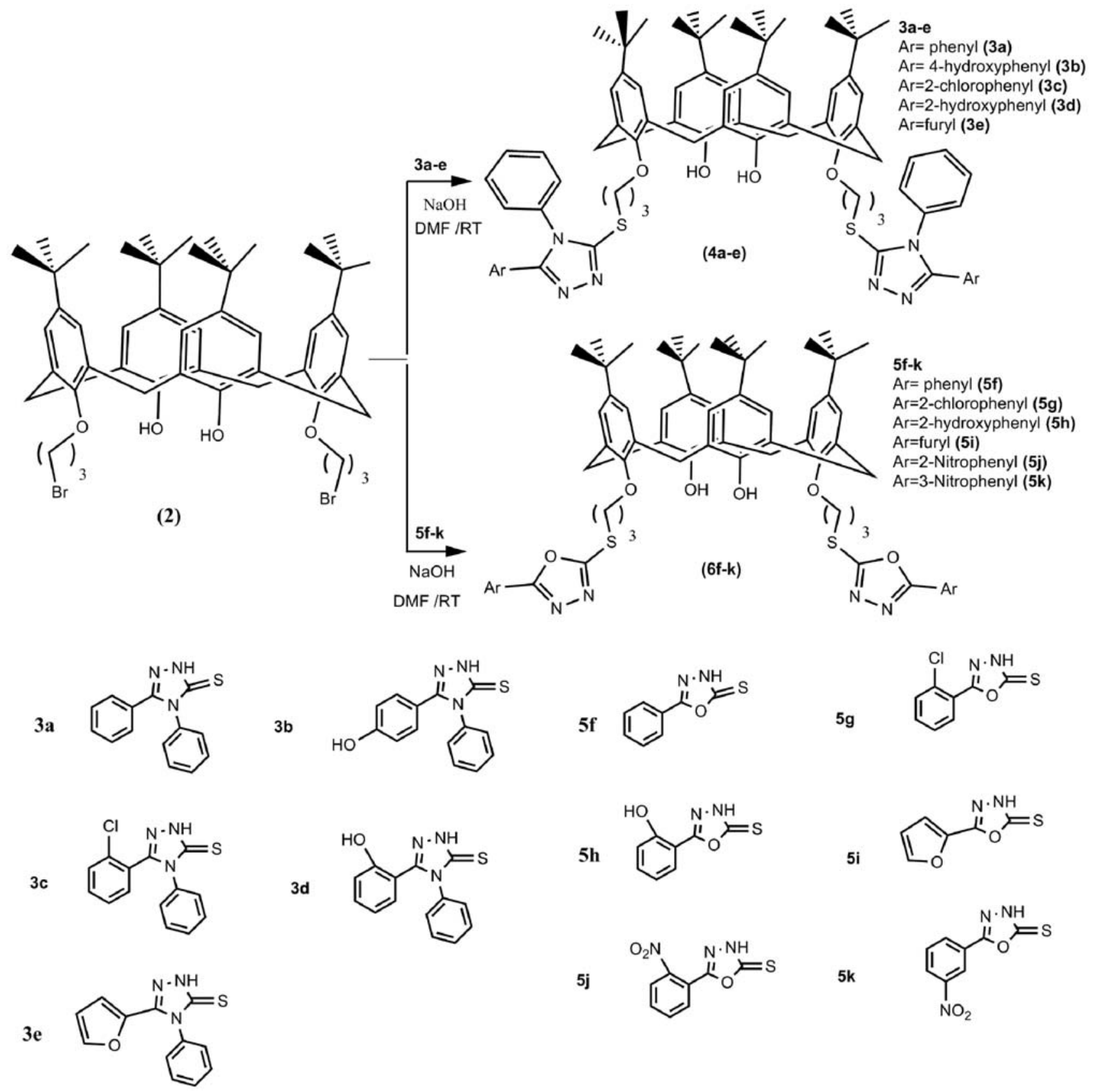

Scheme 3. Synthesis of 1,2,4-triazole-5-thiones and 1,3,4-oxadiazole-5-thiones linked to tetra-tert-butyl calix[4] arene.

the conformation changes of calix[4]arene (2) controlled by regulating the temperature and secondly the derivatization of calix[4]arenes with bulky groups at phenolic oxygen atoms usually leads to conformationally rigid compounds.

The conformations of calix[4]arene can be deduced from the ${ }^{13} \mathrm{C}$-NMR chemical shifts of the methylene groups connecting each pair of aromatic rings. ${ }^{48}$ The structures of the new compounds were fully confirmed by their FT-IR, ${ }^{1} \mathrm{H}-\mathrm{NMR},{ }^{13} \mathrm{C}-\mathrm{NMR}$, DEPT, and mass spectra (ESI-MS).

\section{Experimental}

\section{1. General}

The melting points of all compounds were recorded on a Philip Harris C4954718 apparatus without calibration. IR and ${ }^{1} \mathrm{H}$ - and ${ }^{13} \mathrm{C}$-NMR spectra were recorded on Thermo Nicolet Nexus 670 FT-IR and Bruker Avance 300 $\mathrm{MHz}$ spectrometers, respectively. ESI-MS measurements were recorded on a Finnigan MAT 95 double-focusing sector-field mass spectrometer. Thin layer chromatography (TLC) analyses were carried out on silica gel pla- 
tes. All chemicals were purchased from Merck (Tehran, Iran) and used as received by standard procedures. All of the instruments, chemicals and solvents were dried according to standard methods. Freshly distilled solvents were used throughout, and anhydrous solvents were dried according to the method reported by Perrin and Armarego. ${ }^{49}$ Microanalyses were performed on a Leco Analyzer 932.

\section{2. General Procedure for the Synthesis of 3a-e:}

Equimolar quantities of carboxylic acid hydrazides $1 \mathbf{a}-\mathbf{k}(0.01 \mathrm{~mol})$ and phenyl isothiocyanate $(1.35 \mathrm{~g}, 0.01$ $\mathrm{mol}$ ) in appropriate amount of absolute ethanol were refluxed for 6-8 h. The formed precipitate of 1-substituted4-phenylthiosemicarbazides was filtered, and then a suspension of $0.04 \mathrm{~g}$ thiosemicarbazides in sodium hydroxide $0.4 \mathrm{~g}(0.01 \mathrm{~mol}$, as a $2 \mathrm{~N}$ solution) was refluxed for $6 \mathrm{~h}$. The reaction mixture was allowed to cool to the room temperature and was then adjusted to $\mathrm{pH} 6$ with diluted hydrochloric acid. The formed precipitate was then filtered, washed with water, dried and recrystallized from ethanol to give 1,2,4-triazole-5-thiones 3a-e in 65-75\% yields. The following compounds were prepared by an analogous procedure. ${ }^{34-36}$

\section{General Procedure for the Synthesis of 5f-k:}

Into a stirred solution of carboxylic acid hydrazides $\mathbf{1 a}-\mathbf{k}$ $(0.1 \mathrm{~mol})$ and $\mathrm{KOH}(5.61 \mathrm{~g}, 0.1 \mathrm{~mol})$ in absolute ethanol $(50 \mathrm{~mL}), \mathrm{CS}_{2}(15.2 \mathrm{~g}, 0.2 \mathrm{~mol})$ was added and the mixture was refluxed for $6 \mathrm{~h}$, until the evolution of hydrogen sulfide has ceased. The reaction mixture was cooled to the room temperature and diluted with water. On acidification with dilute hydrochloric acid, the desired oxadiazole was precipitated. The precipitate was filtered, washed thoroughly with cold water and recrystallized from ethanol to give 1,3,4-oxadiazole-5-thiones $\mathbf{5 f - k}$ in $65-70 \%$ yields. The following compounds were prepared by an analogous procedure. ${ }^{37-38}$

\section{Synthesis of Tetra-tert-butylcalix[4]arene (1):}

It was prepared by Gutsche method ${ }^{29}$ as white crystals. Yield 3.97g (62\%), mp 340-343 ${ }^{\circ} \mathrm{C}$; IR (KBr) v $/ \mathrm{cm}^{-1}$ : 3158, 2959, 2905, 2868, 2744, 1604, 1482, 1201, 871, 817, 783, 743, 698; ${ }^{1} \mathrm{H}$ NMR (300 MHz, $\left.\mathrm{CDCl}_{3}\right): \delta_{\mathrm{H}} 1.22$ $\left(\mathrm{s}, 36 \mathrm{H}, 4 \times \mathrm{C}\left(\mathrm{CH}_{3}\right)_{3}\right), 3.5(\mathrm{~d}, 4 \mathrm{H}, J=13.8 \mathrm{~Hz}$, $\left.\mathrm{ArCH}_{2} \mathrm{Ar}\right), 4.26$ (d, 4H, J = $13.8 \mathrm{~Hz}, \mathrm{ArCH}_{2} \mathrm{Ar}$ ), 7.06 (s, $8 \mathrm{H}, \mathrm{ArH}), 10.34(\mathrm{~s}, 4 \mathrm{H}, 4 \times \mathrm{OH}) ;{ }^{13} \mathrm{C}$ NMR $(300 \mathrm{MHz}$, $\left.\mathrm{CDCl}_{3}\right): \delta_{\mathrm{c}} 31.39,32.60,34.75,125.93,127.68,144.36$, 146.66 .

\section{Synthesis of $5,11,17,23$-Tetra-tert-butyl-25,27-bis} (3-bromopropoxy)-26,28-dihydroxycalix[4]arene (2):

It was also prepared with a cone conformation according

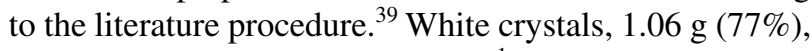
mp 288-290 ${ }^{\circ} \mathrm{C}$; IR (KBr) v/cm ${ }^{-1}: 3406,3044,2959$,
2904, 2867, 1598, 1485, 1201, 1023, 872; ${ }^{1} \mathrm{H}$ NMR (300 $\left.\mathrm{MHz}, \mathrm{CDCl}_{3}\right): \delta_{\mathrm{H}} 1.03\left(\mathrm{~s}, 18 \mathrm{H}, 2 \times \mathrm{C}\left(\mathrm{CH}_{3}\right)_{3}\right), 1.29(\mathrm{~s}$, $\left.18 \mathrm{H}, 2 \times \mathrm{C}\left(\mathrm{CH}_{3}\right)_{3}\right), 2.54\left(\mathrm{t}, 4 \mathrm{H}, J=5.7 \mathrm{~Hz}, 2 \times \mathrm{CH}_{2}\right), 3.36$ $\left(\mathrm{d}, 4 \mathrm{H}, J=12.9 \mathrm{~Hz}, \mathrm{ArCH}_{2} \mathrm{Ar}\right), 4.02(\mathrm{t}, 4 \mathrm{H}, J=6.6 \mathrm{~Hz}, 2$ $\left.\times \mathrm{CH}_{2} \mathrm{Br}\right), 4.13\left(\mathrm{t}, 4 \mathrm{H}, J=5.4 \mathrm{~Hz}, 2 \times \mathrm{OCH}_{2}\right), 4.28(\mathrm{~d}, 4 \mathrm{H}$, $\left.J=12.9 \mathrm{~Hz}, \mathrm{ArCH}_{2} \mathrm{Ar}\right), 6.89(\mathrm{~s}, 4 \mathrm{H}, \mathrm{ArH}), 7.06(\mathrm{~s}, 4 \mathrm{H}$, $\mathrm{ArH}), 7.66(\mathrm{~s}, 2 \mathrm{H}, 2 \times \mathrm{OH}) ;{ }^{13} \mathrm{C} \mathrm{NMR}\left(300 \mathrm{MHz}, \mathrm{CDCl}_{3}\right)$ : $\delta_{\mathrm{c}} 30.36,31.05,31.69,31.88,33.51,33.82,34.04,73.35$, 125.21, 126.71, 127.54, 132.79, 141.69, 147.27, 149.17, 150.59 .

General Procedure for the Synthesis of Tetra-tertbutylcalix[4]arene Derivatives 4a-e and 6f-k:

1,2,4-Triazole or 1,3,4-oxadiazole derivatives $(1 \mathrm{mmol})$ $3 \mathbf{a}-\mathbf{e}$ or $\mathbf{5 f}-\mathbf{k}$ was added to a solution of $0.04 \mathrm{~g}(1 \mathrm{mmol})$ $\mathrm{NaOH}$ in $\mathrm{H}_{2} \mathrm{O}(0.5 \mathrm{~mL})$ and $\mathrm{DMF}(35 \mathrm{~mL})$. The mixture was stirred at room temperature for $30 \mathrm{~min}, 0.223 \mathrm{~g}(0.25$ mmol) of compound 2 was added and stirring at room temperature was continued for further $15 \mathrm{~h}$. The reaction mixture was transferred into cool water and the precipitate was filtered and then recrystallized from methanol, then the precipitate of the compound was submitted to column chromatography $\left(\mathrm{SiO}_{2}\right.$, EtOAc/hexane, 3:8).

Synthesis of 5,11,17,23-Tetra-tert-butyl-25,27-bis(4,5diphenyl-1,2,4-triazole-3-thiapropoxy)-26,28-dihydroxycalix[4]arene (4a):

White crystals; $40 \%$ (0.143 g), mp 160-162 ${ }^{\circ} \mathrm{C}$; IR (KBr) $\mathrm{v} / \mathrm{cm}^{-1}: 3424,2936,1575,1420,1204,1052,763,694$, 639; ${ }^{1} \mathrm{H}$ NMR $\left(300 \mathrm{MHz}, \mathrm{CDCl}_{3}\right): \delta_{\mathrm{H}} 1.03(\mathrm{~s}, 18 \mathrm{H}, 2 \times$ $\left.\mathrm{C}\left(\mathrm{CH}_{3}\right)_{3}\right), 1.29\left(\mathrm{~s}, 18 \mathrm{H}, 2 \times \mathrm{C}\left(\mathrm{CH}_{3}\right)_{3}\right), 2.56(\mathrm{~m}, 4 \mathrm{H}, 2 \times$ $\left.\mathrm{CH}_{2}\right), 3.31\left(\mathrm{~d}, 4 \mathrm{H}, J=12.6 \mathrm{~Hz}, \mathrm{ArCH}_{2} \mathrm{Ar}\right), 3.79(\mathrm{~m}, 4 \mathrm{H}, 2$ $\left.\times \mathrm{SCH}_{2}\right), 4.12\left(\mathrm{~m}, 4 \mathrm{H}, 2 \times \mathrm{OCH}_{2}\right), 4.24(\mathrm{~d}, 4 \mathrm{H}, J=12.8$ $\mathrm{Hz}, \mathrm{ArCH}_{2} \mathrm{Ar}$ ), 6.88 (s, 4H, ArH), 7.05 (s, 4H, ArH), 7.24-7.27 (m, 10H, ArH), 7.41-7.47 (m, 10H, ArH), 7.78 $(\mathrm{s}, 2 \mathrm{H}, 2 \times \mathrm{OH}) ;{ }^{13} \mathrm{C}$ NMR $\left(300 \mathrm{MHz}, \mathrm{CDCl}_{3}\right): \delta_{\mathrm{c}} 29.67$, 30.16, 31.37, 31.77, 32.40, 74.34, 125.92, 125.97, 126.42, $126.45,127.87,128.54,128.78,129.89,147.18,149.36$, 150.67, 152.67, 152.77, 154.87; DEPT $135^{\circ}(125 \mathrm{MHz}$, $\left.\mathrm{CD}_{2} \mathrm{Cl}_{2}\right) \delta(\mathrm{ppm}): 130.25,130.12,129.89,128.78,128.53$, 127.86, 126.44, 125.91, 74.35, 32.44, 31.72, 31.32, 30.15, 29.65; ESI-MS: $m / z=1235.62[\mathrm{M}+\mathrm{H}]$, Calcd: C, 75.35; H, 6.67; N, 7.12; S, 5.44\%. Found: C, 75.29; H, 6.61; N, $7.17 ; \mathrm{S}, 5.33 \%$.

Synthesis of 5,11,17,23-Tetra-tert-butyl-25,27-bis(5(4-hydroxyphenyl)-4-phenyl-1,2,4-triazol-3-thiapropoxy)-26,28-dihydroxycalix[4]arene (4b):

White crystals; $46 \%$ (0.151 g), mp $185-187^{\circ} \mathrm{C}$; IR ( $\left.\mathrm{KBr}\right)$ $\mathrm{v} / \mathrm{cm}^{-1}: 3409,3048,2952,1599,1554,1477,1305,1208$, $1020,925,878,752,693,630,557 ;{ }^{1} \mathrm{H}$ NMR $(300 \mathrm{MHz}$, $\left.\mathrm{CDCl}_{3}\right): \delta_{\mathrm{H}} 1.04\left(\mathrm{~s}, 18 \mathrm{H}, 2 \times \mathrm{C}\left(\mathrm{CH}_{3}\right)_{3}\right), 1.31(\mathrm{~s}, 18 \mathrm{H}, 2 \times$ $\left.\mathrm{C}\left(\mathrm{CH}_{3}\right)_{3}\right), 2.49\left(\mathrm{~m}, 4 \mathrm{H}, 2 \times \mathrm{CH}_{2}\right), 3.33(\mathrm{~d}, 4 \mathrm{H}, J=12.9$ $\left.\mathrm{Hz}, \mathrm{ArCH}_{2} \mathrm{Ar}\right), 3.61$ (m, 4H, $\left.2 \times \mathrm{SCH}_{2}\right), 4.08(\mathrm{~m}, 4 \mathrm{H}, 2 \times$ $\left.\mathrm{OCH}_{2}\right), 4.23\left(\mathrm{~d}, 4 \mathrm{H}, J=12.6 \mathrm{~Hz}, \mathrm{ArCH}_{2} \mathrm{Ar}\right), 6.88(\mathrm{~s}, 4 \mathrm{H}$, ArH), 6.97 (t, $2 \mathrm{H}, J=7.2 \mathrm{~Hz}, \mathrm{ArH}), 7.06$ (s, 4H, ArH), 
$7.24(\mathrm{~s}, 2 \mathrm{H}, 2 \times \mathrm{OH}) 7.26(\mathrm{t}, 2 \mathrm{H}, J=7.5 \mathrm{~Hz}, \mathrm{ArH}), 7.36$ (d, $4 \mathrm{H}, J=7.2 \mathrm{~Hz}, \mathrm{ArH}), 7.49-7.56(\mathrm{~m}, 10 \mathrm{H}, \mathrm{ArH}+2 \times$ $\mathrm{OH}), 7.74(\mathrm{~s}, 2 \mathrm{H}, 2 \times \mathrm{OH}) ;{ }^{13} \mathrm{C} \mathrm{NMR}\left(300 \mathrm{MHz}, \mathrm{CDCl}_{3}\right)$ : $\delta_{c} 29.98,30.23,31.36,31.72,32.44,73.38,117.43$, $117.49,122.01,125.47,125.96,128.09,130.45,130.64$, $130.88,147.09,149.43,150.65,151.51$; DEPT $135^{\circ}(125$ $\left.\mathrm{MHz}, \mathrm{CD}_{2} \mathrm{Cl}_{2}\right) \delta(\mathrm{ppm}): 130.85,130.66,130.40,128.04$, 126.43, 125.91, 122.03, 117.48, 117.42, 74.32, 32.44, 31.73, 31.34, 30.23, 30.00; ESI-MS: $\mathrm{m} / \mathrm{z}=1265.65$ [M-H]; Calcd: C, 73.36; H, 6.49; N, 6.94; S, $5.29 \%$. Found: C, 73.19; H, 6.53; N, 6.83; S, 5.24\%.

Synthesis of 5,11,17,23-Tetra-tert-butyl-25,27-bis(5(2-chlorophenyl)-4-phenyl-1,2,4-triazole-3-thiapropoxy)-26,28-dihydroxycalix [4] arene $(4 \mathrm{c})$ :

White crystals; $0.14 \mathrm{~g}(40 \%), \mathrm{mp} 250-252{ }^{\circ} \mathrm{C}$; IR (KBr) $\mathrm{v} / \mathrm{cm}^{-1}: 3432,3033,2956,1595,1485,1430,1301,1200$, 1123, 1031, 873, 763, 964, 600; ${ }^{1} \mathrm{H}$ NMR $(300 \mathrm{MHz}$, $\left.\mathrm{CDCl}_{3}\right): \delta_{\mathrm{H}} 1.02\left(\mathrm{~s}, 18 \mathrm{H}, 2 \times \mathrm{C}\left(\mathrm{CH}_{3}\right)_{3}\right), 1.34(\mathrm{~s}, 18 \mathrm{H}, 2 \times$ $\left.\mathrm{C}\left(\mathrm{CH}_{3}\right)_{3}\right), 2.59\left(\mathrm{~m}, 4 \mathrm{H}, 2 \times \mathrm{CH}_{2}\right), 3.29(\mathrm{~d}, 4 \mathrm{H}, J=12.9$ $\left.\mathrm{Hz}, \mathrm{ArCH}_{2} \mathrm{Ar}\right), 3.85\left(\mathrm{~m}, 4 \mathrm{H}, 2 \times \mathrm{SCH}_{2}\right), 4.12(\mathrm{~m}, 4 \mathrm{H}, 2 \times$ $\left.\mathrm{OCH}_{2}\right), 4.2\left(\mathrm{~d}, 4 \mathrm{H}, J=12.6 \mathrm{~Hz}, \mathrm{ArCH}_{2} \mathrm{Ar}\right), 6.85(\mathrm{~s}, 4 \mathrm{H}$, ArH), 7.04 (s, 4H, ArH), 7.14-7.15 (m, 4H, ArH), 7.27-7.41 (m, 14H, ArH), $7.73(\mathrm{~s}, 2 \mathrm{H}, 2 \times \mathrm{OH}) ;{ }^{13} \mathrm{C} \mathrm{NMR}$ (300 MHz, $\mathrm{CDCl}_{3}$ ): $\delta_{\mathrm{c}} 29.65,31.01,31.12,31.68,31.72$, $33.81,73.32,125.09,125.69,126.70,126.87,127.49$, $129.52,129.92,132.75,133.37,141.49,147.06,149.39$, 150.71, 152.27, 153.28; DEPT $135^{\circ}\left(125 \mathrm{MHz}, \mathrm{CD}_{2} \mathrm{Cl}_{2}\right)$ : $\delta 147.05,141.49,133.37,132.74,129.91,129.53,127.41$, 126.88, 125.69, 125.06, 73.32, 33.83, 31.63, 31.14, 31.03, 29.67; ESI-MS: $m / z=1305.46[\mathrm{M}+\mathrm{H}]$; Calcd: C, 71.19; H, 6.14; N, 6.73; S, 5.14\%. Found: C, 70.85; H, 6.07; N, $6.79 ; \mathrm{S}, 5.10 \%$.

Synthesis of 5,11,17,23-Tetra-tert-butyl-25,27-bis(5(2-hydroxy phenyl)-4-phenyl-1,2,4-triazol-3-thiapropoxy)-26,28-dihydroxycalix[4]arene (4d):

White crystals; $0.14 \mathrm{~g}(0.43 \%), \mathrm{mp} 190-192{ }^{\circ} \mathrm{C}$; IR (KBr) $\mathrm{v} / \mathrm{cm}^{-1}: 3352,3051,2952,1589,1481,1293,1247$, 1204, 1023, 747, 699, 609; ${ }^{1} \mathrm{H}$ NMR (300 MHz, $\left.\mathrm{CDCl}_{3}\right)$ : $\delta_{\mathrm{H}} 1.04\left(\mathrm{~s}, 18 \mathrm{H}, 2 \times \mathrm{C}\left(\mathrm{CH}_{3}\right)_{3}\right), 1.29\left(\mathrm{~s}, 18 \mathrm{H}, 2 \times \mathrm{C}\left(\mathrm{CH}_{3}\right)_{3}\right)$, $2.53\left(\mathrm{~m}, 4 \mathrm{H}, 2 \times \mathrm{CH}_{2}\right), 3.31(\mathrm{~d}, 4 \mathrm{H}, J=12.9 \mathrm{~Hz}, \mathrm{Ar}-$ $\left.\mathrm{CH}_{2} \mathrm{Ar}\right), 3.64-3.80\left(\mathrm{~m}, 4 \mathrm{H}, 2 \times \mathrm{SCH}_{2}\right), 4.09(\mathrm{~m}, 4 \mathrm{H}$, $\left.\mathrm{OCH}_{2}\right), 4.19\left(\mathrm{~d}, 4 \mathrm{H}, J=12.6 \mathrm{~Hz}, \mathrm{ArCH}_{2} \mathrm{Ar}\right), 6.51-6.57$ (m, 2H,ArH), 6.88 (s, 4H, ArH), 7.04 (s, 4H, ArH), 7.09 (s, 2H, $2 \times \mathrm{OH}), 7.17-7.36(\mathrm{~m}, 8 \mathrm{H}, \mathrm{ArH}), 7.49-7.58(\mathrm{~m}$, $8 \mathrm{H}, \mathrm{ArH}), 7.77(\mathrm{~s}, 2 \mathrm{H}, 2 \times \mathrm{OH}) ;{ }^{13} \mathrm{C}$ NMR $(300 \mathrm{MHz}$, $\left.\mathrm{CDCl}_{3}\right): \delta_{\mathrm{c}} 29.66,30.03,30.07,30.29,31.32,31.72$, $32.42,74.19,74.45,117.43,117.91,118.81,122.04$, $125.90,125.95,126.04,126.45,126.94,128.09,128.17$, $129.39,130.71,130.85,131.03,131.54,146.49,149.38$, 151.38, 153.30; DEPT $135^{\circ}\left(125 \mathrm{MHz}, \mathrm{CD}_{2} \mathrm{Cl}_{2}\right): \delta$ $131.00,130.90,130.83,130.71,129.39,128.14,128.02$, $126.45,126.04,125.94,125.90,122.04,118.82,117.98$, $117.43,74.45,74.18,32.42,31.72,31.33,30.29,30.07$, 30.00, 29.67; ESI-MS: $m / z=1266.63$ [M-H]; Calcd: C,
73.36; H, 6.49; N, 6.94; S, 5.29\%. Found: C, 73.25; H, $6.54 ; \mathrm{N}, 6.90 ; \mathrm{S}, 5.31 \%$.

Synthesis of compound : 5,11,17,23-Tetra-tert-butyl25,27-bis(5-(furan-2-yl)-4-phenyl-1,2,4-triazole 3-thiapropoxy)-26,28-dihydroxycalix[4]arene (4e):

White crystals; $0.113 \mathrm{~g}(43 \%), \mathrm{mp} 195-197{ }^{\circ} \mathrm{C}$; IR ( $\left.\mathrm{KBr}\right)$ $\mathrm{v} / \mathrm{cm}^{-1}$ : 3382, 2936, 2870, 1600, 1477, 1204, 1021, 725, 696, 604; ${ }^{1} \mathrm{H}$ NMR $\left(300 \mathrm{MHz}, \mathrm{CDCl}_{3}\right): \delta_{\mathrm{H}} 1.01(\mathrm{~s}, 18 \mathrm{H}, 2$ $\left.\times \mathrm{C}\left(\mathrm{CH}_{3}\right)_{3}\right), 1.27\left(\mathrm{~s}, 18 \mathrm{H}, 2 \times \mathrm{C}\left(\mathrm{CH}_{3}\right)_{3}\right), 2.49(\mathrm{~m}, 4 \mathrm{H}, 2 \times$ $\left.\mathrm{CH}_{2}\right), 3.28\left(\mathrm{~d}, 4 \mathrm{H}, J=13 \mathrm{~Hz}, \mathrm{ArCH}_{2} \mathrm{Ar}\right), 3.78(\mathrm{t}, 4 \mathrm{H}, J=$ $\left.6.6 \mathrm{~Hz}, 2 \times \mathrm{SCH}_{2}\right), 4.07\left(\mathrm{t}, 4 \mathrm{H}, J=5.4 \mathrm{~Hz}, 2 \times \mathrm{OCH}_{2}\right), 4.1$ $\left(\mathrm{d}, 4 \mathrm{H}, J=15.6 \mathrm{~Hz}, \mathrm{ArCH}_{2} \mathrm{Ar}\right), 6.15(\mathrm{~d}, 2 \mathrm{H}, J=3.39 \mathrm{~Hz}$, $\mathrm{H}$ furyl), 6.32 (t, $2 \mathrm{H}, J=1.8 \mathrm{~Hz}, \mathrm{H}$ furyl), $6.85(\mathrm{~s}, 4 \mathrm{H}$, ArH), 7.02 (s, 4H, ArH), 7.27-7.31 (m, 3H, ArH), 7.4 (d, 2H, H furyl), 7.53-7.55 (m, 7H, ArH), 7.69 (s, 2H, $2 \times$ $\mathrm{OH}) ;{ }^{13} \mathrm{C}$ NMR $\left(300 \mathrm{MHz}, \mathrm{CD}_{2} \mathrm{Cl}_{2}\right): \delta_{\mathrm{c}} 29.33,29.80$, $31.68,31.92,32.16,33.99,73.74,111.15,125.11,125.60$, $127.54,129.95,130.35,132.72,133.67,141.52,141.61$, $143.81,143.94,147.07,147.91,149.37,151.61$; DEPT $135^{\circ}\left(125 \mathrm{MHz}, \mathrm{CDCl}_{3}\right): \delta 133.68,132.73,130.37$, $129.95,127.54,125.61,125.11,111.15,73.74,33.98$, 32.16, 31.92, 31.67, 29.81, 29.33; ESI-MS: $\mathrm{m} / \mathrm{z}=1232.71$ [M+H]; Calcd: C, 72.51; H, 6.43; N, 7.25, S, 5.53\%. Found: C, 75.40; H, 6.39; N, 7.21; S, 5.43\%.

Synthesis of $5,11,17,23$-Tetra-tert-butyl-25,27-bis (5-(phenyl)-1,3,4-oxadiazole-2-thiapropoxy)-26,28dihydroxycalix[4]arene (6f):

White crystals; $0.145 \mathrm{~g}(53 \%), \mathrm{mp} 150-152{ }^{\circ} \mathrm{C}$; IR (KBr) $\mathrm{v} / \mathrm{cm}^{-1}$ : 3386, 2951, 1599, 1474, 1197, 1037, 879, 696, 473; ${ }^{1} \mathrm{H}$ NMR $\left(300 \mathrm{MHz}, \mathrm{CDCl}_{3}\right): \delta_{\mathrm{H}} 1.01(\mathrm{~s}, 18 \mathrm{H}$, $\left.2 \mathrm{C}\left(\mathrm{CH}_{3}\right)_{3}\right), 1.29\left(\mathrm{~s}, 18 \mathrm{H}, 2 \mathrm{C}\left(\mathrm{CH}_{3}\right)_{3}\right), 2.60\left(\mathrm{~m}, 4 \mathrm{H}, 2 \mathrm{CH}_{2}\right)$, $3.37\left(\mathrm{~d}, 4 \mathrm{H}, J=12.9 \mathrm{~Hz}, \mathrm{ArCH}_{2} \mathrm{Ar}\right), 3.91(\mathrm{~m}, 4 \mathrm{H}$, $\left.2 \mathrm{SCH}_{2}\right), 4.18\left(\mathrm{~m}, 4 \mathrm{H}, 2 \times \mathrm{OCH}_{2}\right), 4.27(\mathrm{~d}, 4 \mathrm{H}, J=12.6$ $\left.\mathrm{Hz}, \mathrm{ArCH}_{2} \mathrm{Ar}\right), 6.87$ (s, 4H, ArH), 7.07 (s, 4H, ArH), 7.45-7.50 (m, 6H, ArH), 7.63 (s, 2H, 2OH), 7.88-8.00 $(\mathrm{m}, 4 \mathrm{H}, \mathrm{ArH}) ;{ }^{13} \mathrm{C} \mathrm{NMR}\left(300 \mathrm{MHz}, \mathrm{CDCl}_{3}\right): \delta_{\mathrm{c}} 29.63$, 29.93, 30.43, 30.73, 31.15, 31.27, 32.37, 32.42, 33.83, $53.23,53.32,73.35,73.55,125.55,125.78,126.09$, $126.42,127.57,129.58,130.53,132.21,132.72,141.77$, 149.18, 150.60; DEPT $135^{\circ}\left(125 \mathrm{MHz}, \mathrm{CD}_{2} \mathrm{Cl}_{2}\right): \delta 132.72$, $132.22,130.54,129.53,127.55,126.47,126.05,125.78$, $125.52,73.55,73.35,53.33,53.35,33.87,32.43,32.33$, $31.24,31.19,30.77,30.43,29.45$, 29.66; ESI-MS: $\mathrm{m} / \mathrm{z}=$ 1084.48 [M-H]; Calcd: C, 72.34; H, 6.66; N, 5.44; S, $6.23 \%$. Found: C, 72.19; H, 6.57; N, 7.40; S, 6.30\%.

Synthesis of 5,11,17,23-Tetra-tert-butyl-25,27-bis(5(2-chlorophenyl)-1,3,4-oxadiazole-2-thiapropoxy)26,28-dihydroxycalix[4]arene (6g):

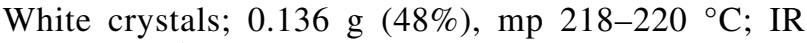
(KBr) $v / \mathrm{cm}^{-1}: 3355,2949,1597,1470,1293,1191,1095$, 1031, 884, 731, 649; ${ }^{1} \mathrm{H}$ NMR $\left(300 \mathrm{MHz}, \mathrm{CDCl}_{3}\right): \delta_{\mathrm{H}}$ $1.01\left(\mathrm{~s}, 18 \mathrm{H}, 2 \mathrm{C}\left(\mathrm{CH}_{3}\right)_{3}\right), 1.29\left(\mathrm{~s}, 18 \mathrm{H}, 2 \mathrm{C}\left(\mathrm{CH}_{3}\right)_{3}\right), 2.61$ $\left(\mathrm{m}, 4 \mathrm{H}, 2 \mathrm{CH}_{2}\right), 3.37\left(\mathrm{~d}, 4 \mathrm{H}, J=13.2 \mathrm{~Hz}, \mathrm{ArCH}_{2} \mathrm{Ar}\right)$, 
3.91-3.93 (m, 4H, $\left.2 \mathrm{SCH}_{2}\right), 4.18\left(\mathrm{~m}, 4 \mathrm{H}, 2 \mathrm{OCH}_{2}\right), 4.26$ (d, $\left.4 \mathrm{H}, J=12.6 \mathrm{~Hz}, \mathrm{ArCH}_{2} \mathrm{Ar}\right), 6.88$ (s, 4H, ArH), 7.07 (s, 4H, ArH), 7.34-7.42 (m, 4H, ArH), 7.50 (d, 2H, ArH), $7.66(\mathrm{~s}, 2 \mathrm{H}, 2 \mathrm{OH}), 7.91(\mathrm{~d}, 2 \mathrm{H}, \mathrm{ArH}) ;{ }^{13} \mathrm{C}$ NMR $(300$ $\left.\mathrm{MHz}, \mathrm{CDCl}_{3}\right): \delta_{\mathrm{c}} 29.71,29.78,30.65,30.88,30.95$, $31.16,31.61,32.08,33.89,53.48,53.85,73.33,73.45$, $125.78,125.85,126.06,126.22,126.55,130.31,131.55$, $132.65,141.77,147.29,149.19,150.54$; DEPT $135^{\circ}(125$ $\left.\mathrm{MHz}, \mathrm{CD}_{2} \mathrm{Cl}_{2}\right): \delta 132.67,131.54,130.33,126.57$, $126.25,126.08,125.80,125.75,73.46,73.35,53.87$, 53.43, 33.83, 32.04, 31.69, 31.17, 30.93, 30.85, 30.66, 29.80, 29.70; ESI-MS: $m / z=1138.33$ [M-H]; Calcd: C, 67.81; H, 6.06; N, 5.10; S, 5.84\%. Found: C, 67.69; H, $5.95 ; \mathrm{N}, 5.14 ; \mathrm{S}, 5.78 \%$.

Synthesis of 5,11,17,23-Tetra-tert-butyl-25,27-bis(5(2-hydroxy phenyl)-1,3,4-oxadiazole-2-thiapropoxy)26,28-dihydroxycalix[4]arene (6h):

White crystals; $0.138 \mathrm{~g}$ (49\%), mp $240-242{ }^{\circ} \mathrm{C}$; IR (KBr) $v / \mathrm{cm}^{-1}: 3393,2951,1743,1595,1476,1295$, 1202, 1112, 1024, 911, 734, 637, 566; ${ }^{1} \mathrm{H}$ NMR (300 $\left.\mathrm{MHz}, \mathrm{CDCl}_{3}\right): \delta_{\mathrm{H}} 1.04\left(\mathrm{~s}, 18 \mathrm{H}, 2 \mathrm{C}\left(\mathrm{CH}_{3}\right)_{3}\right), 1.29(\mathrm{~s}, 18 \mathrm{H}$, $\left.2 \mathrm{C}\left(\mathrm{CH}_{3}\right)_{3}\right), 2.54-2.61\left(\mathrm{~m}, 4 \mathrm{H}, 2 \mathrm{CH}_{2}\right), 3.37(\mathrm{~d}, 4 \mathrm{H}, J=$ $\left.12.6 \mathrm{~Hz}, \mathrm{ArCH}_{2} \mathrm{Ar}\right), 3.93-4.13\left(\mathrm{~m}, 4 \mathrm{H}, 2 \mathrm{SCH}_{2}\right)$, $4.18-4.26\left(\mathrm{~m}, 4 \mathrm{H}, 2 \mathrm{OCH}_{2}\right), 4.28(\mathrm{~d}, 4 \mathrm{H}, J=12.9 \mathrm{~Hz}$, $\left.\mathrm{ArCH}_{2} \mathrm{Ar}\right), 6.88-6.93(\mathrm{~m}, 7 \mathrm{H}, \mathrm{ArH}), 6.93-7.07(\mathrm{~m}, 7 \mathrm{H}$, $\mathrm{ArH}), 7.41(\mathrm{~s}, 2 \mathrm{H}, 2 \times \mathrm{OH}), 7.87-7.93(\mathrm{~m}, 4 \mathrm{H}, 2 \mathrm{OH}+$ $\mathrm{ArH}) ;{ }^{13} \mathrm{C}$ NMR $\left(300 \mathrm{MHz}, \mathrm{CDCl}_{3}\right): \delta_{\mathrm{c}} 29.51,29.61$, $30.83,30.95,30.99,31.38,31.97,32.09,33.59,53.44$, $53.64,53.80,73.53,73.66,117.24,119.65,125.58$, $125.67,126.15,126.19,126.51,133.49,147.38,149.12$, 150.48, 157.13, 165.22; DEPT $135^{\circ}\left(125 \mathrm{MHz}, \mathrm{CD}_{2} \mathrm{Cl}_{2}\right)$ : $\delta 133.47,126.43,126.17,126.14,125.63,125.60$, 119.94, 117.20, 73.64, 73.56, 53.80, 53.61, 53.43, 33.53, 32.04, 31.99, 31.32, 30.93, 30.65, 30.81, 29.50, 29.64; ESI-MS: $m / z=1116.51[\mathrm{M}-\mathrm{H}]$; Calcd: C, 70.16; H, 6.46; N, 5.28; S, 6.04\%. Found: C, 69.99; H, 6.40; N, 5.17; S, $6.10 \%$.

Synthesis of 5,11,17,23-Tetra-tert-butyl-25,27-bis (5-(furan-2-yl)-1,3,4-oxadiazole-2-thiapropoxy)-26,28dihydroxycalix [4]arene (6i):

White crystals; $0.161 \mathrm{~g}(60 \%), \mathrm{mp} 140-142{ }^{\circ} \mathrm{C}$; IR (KBr) $\mathrm{v} / \mathrm{cm}^{-1}: 3418,2951,1629,1472,1195,1091,894,754$, 468; ${ }^{1} \mathrm{H}$ NMR $\left(300 \mathrm{MHz}, \mathrm{CDCl}_{3}\right): \delta_{\mathrm{H}} 0.99(\mathrm{~s}, 18 \mathrm{H}$, $\left.2 \mathrm{C}\left(\mathrm{CH}_{3}\right)_{3}\right), 1.32\left(\mathrm{~s}, 18 \mathrm{H}, 2 \times \mathrm{C}\left(\mathrm{CH}_{3}\right)_{3}\right), 2.61(\mathrm{~m}, 4 \mathrm{H}$, $\left.2 \mathrm{CH}_{2}\right), 3.39\left(\mathrm{~d}, 4 \mathrm{H}, J=12.9 \mathrm{~Hz}, \mathrm{ArCH}_{2} \mathrm{Ar}\right), 3.92(\mathrm{~m}, 4 \mathrm{H}$, $\left.2 \mathrm{SCH}_{2}\right), 4.19\left(\mathrm{~m}, 4 \mathrm{H}, 2 \mathrm{OCH}_{2}\right), 4.28(\mathrm{~d}, 4 \mathrm{H}, J=12.9 \mathrm{~Hz}$, $\left.\mathrm{ArCH}_{2} \mathrm{Ar}\right), 6.28$ (m, 2H, H furyl), 6.88 (s, 4H, ArH), 6.92 (d, $2 \mathrm{H}, J=3.3 \mathrm{~Hz}, \mathrm{H}$ furyl), 7.10 (s, 4H, ArH), 7.37 (s, $2 \mathrm{H}, \mathrm{H}$ furyl), 7.80 (s, $2 \mathrm{H}, 2 \mathrm{OH}) ;{ }^{13} \mathrm{C}$ NMR $(300 \mathrm{MHz}$, $\left.\mathrm{CDCl}_{3}\right): \delta_{\mathrm{c}} 29.55,29.59,29.65,29.68,30.91,30.94$, $31.35,31.38,32.04,32.07,53.64,53.67,73.79,112.13$, $113.79,125.43,125.66,126.11,145.65,147.36,149.16$, $158.59,163.65$; DEPT $135^{\circ}\left(125 \mathrm{MHz}, \mathrm{CD}_{2} \mathrm{Cl}_{2}\right): \delta$ $145.68,126.15,125.60,113.74,112.04,73.64,53.79$,
53.61, 32.06, 32.03, 31.35, 31.33, 30.95, 30.93, 29.68, 29.66, 29.56, 29.54; ESI-MS: $m / z=1066.48[\mathrm{M}+\mathrm{H}]$; Calcd: C, 69.02; H, 6.39; N, 5.55; S, 6.35\%. Found: C, $68.85 ; \mathrm{H}, 6.37$; N, 5.27; S, 6.30\%.

Synthesis of 5,11,17,23-Tetra-tert-butyl-25,27-bis(5(2-nitrophenyl)-1,3,4-oxadiazole-2-thiapropoxy)26,28-dihydroxycalix[4]arene $(6 \mathbf{6 j})$ :

White crystals; $0.140 \mathrm{~g}(47 \%), \mathrm{mp} 143-145^{\circ} \mathrm{C}$; IR (KBr) $\mathrm{v} / \mathrm{cm}^{-1}: 3389,2947,1713,1537,1468,1356,1281,1189$, 1110, 1033, 890, 774, 716; ${ }^{1} \mathrm{H}$ NMR (300 MHz, $\mathrm{CDCl}_{3}$ ): $\delta_{\mathrm{H}} 0.99\left(\mathrm{~s}, 18 \mathrm{H}, 2 \mathrm{C}\left(\mathrm{CH}_{3}\right)_{3}\right), 1.29\left(\mathrm{~s}, 18 \mathrm{H}, 2 \mathrm{C}\left(\mathrm{CH}_{3}\right)_{3}\right), 2.57$ $\left(\mathrm{m}, 4 \mathrm{H}, 2 \mathrm{CH}_{2}\right), 3.35\left(\mathrm{~d}, 4 \mathrm{H}, J=12.9 \mathrm{~Hz}, \mathrm{ArCH}_{2} \mathrm{Ar}\right)$, 3.86-3.90 (m, 4H, $\left.2 \mathrm{SCH}_{2}\right), 4.17\left(\mathrm{~m}, 4 \mathrm{H}, 2 \mathrm{OCH}_{2}\right), 4.24$ (d, $\left.4 \mathrm{H}, J=13.3 \mathrm{~Hz}, \mathrm{ArCH}_{2} \mathrm{Ar}\right), 6.86(\mathrm{~s}, 4 \mathrm{H}, \mathrm{ArH}), 7.07$ (m, $4 \mathrm{H}, \mathrm{ArH}), 7.60-7.64(\mathrm{~m}, 4 \mathrm{H}, \mathrm{ArH}), 7.68(\mathrm{~s}, 2 \mathrm{H}, 2 \times \mathrm{OH})$, 7.87-7.93 (m, 4H,ArH); $\left.{ }^{13} \mathrm{C} \mathrm{NMR} \mathrm{(300} \mathrm{MHz,} \mathrm{CDCl}_{3}\right): \delta_{\mathrm{c}}$ $29.63,29.65,29.67,29.69,30.91,30.95,31.36,31.39$, $32.09,32.85,73.55,124.41,124.67,125.63,126.08$, 131.29, 132.42, 133.52, 147.30, 149.16, 162.34, 165.77; DEPT $135^{\circ}\left(125 \mathrm{MHz}, \mathrm{CD}_{2} \mathrm{Cl}_{2}\right): \delta 133.09,132.45$, $131.26,126.14,125.60,124.65,124.52,73.65,32.03$, 32.01, 31.34, 31.33, 30.94, 30.92, 29.68, 29.67, 29.64, 29.62; ESI-MS: $\mathrm{m} / \mathrm{z}=1176.49[\mathrm{M}+\mathrm{H}]$; Calcd: C, 66.53; H, 5.94; N, 7.51; S, 5.73\%. Found: C, 66.35; H, 5.80; N, 7.37; S, 5.86\%.

Synthesis of 5,11,17,23-Tetra-tert-butyl-25,27-bis(5(3-nitrophenyl)-1,3,4-oxadiazole-2-thiapropoxy)26,28-dihydroxycalix[4]arene (6k):

White crystals; $0.134 \mathrm{~g}(45 \%), \mathrm{mp} 138-140{ }^{\circ} \mathrm{C}$; IR (KBr) $\mathrm{v} / \mathrm{cm}^{-1}: 3332,2945,1723,1537,1470,1354,1289,1196$, 1111, 1028, 928, 718, 641, 581; ${ }^{1} \mathrm{H}$ NMR $(300 \mathrm{MHz}$, $\left.\mathrm{CDCl}_{3}\right): \delta_{\mathrm{H}} 0.97\left(\mathrm{~s}, 18 \mathrm{H}, 2 \mathrm{C}\left(\mathrm{CH}_{3}\right)_{3}\right), 1.31(\mathrm{~s}, 18 \mathrm{H}$, $\left.2 \mathrm{C}\left(\mathrm{CH}_{3}\right)_{3}\right), 2.58\left(\mathrm{~m}, 4 \mathrm{H}, 2 \mathrm{CH}_{2}\right), 3.37(\mathrm{~d}, 4 \mathrm{H}, J=13.2 \mathrm{~Hz}$, $\left.\mathrm{ArCH}_{2} \mathrm{Ar}\right), 3.87\left(\mathrm{~m}, 4 \mathrm{H}, 2 \mathrm{SCH}_{2}\right), 4.18\left(\mathrm{~m}, 4 \mathrm{H}, 2 \mathrm{OCH}_{2}\right)$, $4.25\left(\mathrm{~d}, 4 \mathrm{H}, J=12.9 \mathrm{~Hz}, \mathrm{ArCH}_{2} \mathrm{Ar}\right), 6.86(\mathrm{~s}, 4 \mathrm{H}, \mathrm{ArH})$, 7.08 (s, 4H, ArH), 7.55-7.59 (m, 4H, ArH), 7.67 (s, 2H, 2OH $), 7.85-7.92(\mathrm{~m}, 4 \mathrm{H}, \mathrm{ArH}) ;{ }^{13} \mathrm{C}$ NMR $(300 \mathrm{MHz}$, $\left.\mathrm{CDCl}_{3}\right): \delta_{\mathrm{c}} 29.66,30.13,30.73,31.18,31.63,32.21$, $32.23,33.97,73.35,118.32,123.93,124.71,125.12$, 125.52, 125.86, 126.13, 130.82, 133.69, 141.84, 147.32, 149.16, 162.32, 165.78; DEPT $135^{\circ}\left(125 \mathrm{MHz}, \mathrm{CD}_{2} \mathrm{Cl}_{2}\right)$ : $\delta 133.69,130.82,126.13,125.86,125.52,125.13,124.72$, $123.93,118.35,73.35,33.98,32.23,32.21,31.64,31.18$, 30.74, 30.12, 29.68; ESI-MS: $m / z=1174.5[\mathrm{M}-\mathrm{H}]$; Calcd: C, 66.53; H, 5.94; N, 7.51; S, 5.73\%. Found: C, $66.28 ; \mathrm{H}, 5.79 ; \mathrm{N}, 7.29 ; \mathrm{S}, 5.98 \%$.

\section{Conclusion}

In summary, a series of new tetra-tert-butylcalix[4]arene derivatives $\mathbf{4 a}-\mathbf{e}$ and $\mathbf{6} \mathbf{f}-\mathbf{k}$ were prepared by reaction of 1,2,4-triazole-5-thione 3a-e and 1,3,4-oxadiazole-5-thione $\mathbf{5 f - k}$ moieties with calix[4]arene (2) in pre- 
sence of sodium hydroxide at room temperature. The new calix[4]arene are reported in this article may have biological and pharmaceutical activities.

\section{Acknowledgments}

The authors are grateful to Urmia University for providing a fellowship for the present work and thanks are also given to Prof. Dr. Joachim Thiem from Hamburg University for the ESI-MS and DEPT measurements.

\section{References}

1. E. da Silva, A. N. Lazar, A. W. Coleman, J. Drug. Del. Sci. Tech. 2004, 14, 3-20. http://dx.doi.org/10.1016/S1773-2247(04)50001-1

2. Â. de Fátima, S. A. Fernadez, A. A. Sabino, Curr. Drug. Discov. Technol. 2009, 6, 151-170. http://dx.doi.org/10.2174/157016309788488302

3. R. V. Rodik, V. I. Boyko, V. I. Kalchenko, Curr. Med. Chem. 2009, 16, 1630-1655. http://dx.doi.org/10.2174/092986709788186219

4. B. Mokhtari, K. Pourabdollah, J. Incl. Phenom. Macrocyl. Chem. 2012, 73, 1-15. http://dx.doi.org/10.1007/s10847-011-0062-z

5. M. D. Shah, Y. K. Agrawal, J. Sci. Ind. Res. India. 2012, 71, 21-26.

6. B. Mokhtari, K. Pourabdollah, Drug. Chem. Toxicol, 2013, 36, 119-132. http://dx.doi.org/10.3109/01480545.2011.653490

7. R. Martinez-Manez, F. Sancenon, Chem. Rev. 2003, 103, 4419-4476. http://dx.doi.org/10.1021/cr010421e

8. R. Metivier, I. Leray, B. Valeur, Chem. Eur. J. 2004, 10, 4480-4490. http://dx.doi.org/10.1002/chem.200400259

9. A. Casnati, M. Fabbi, N. Pelizzi, A. Pochini, F. Sansone, R. Ungaro, E. Di Modugno, G. Tarzia, Bioorg. Med. Chem. Lett. 1996, 6, 2699-2704. http://dx.doi.org/10.1016/S0960-894X(96)00493-3

10. E. J. Kim, J. I. Choe, S. Chang, Tetrahedron Lett. 2003, 44, 5299-5302. http://dx.doi.org/10.1016/S0040-4039(03)01211-5

11. D. H. Williams, B. Bardsley, Angew. Chem. Int. Ed. Engl. 1999, 38, 1172-1193. http://dx.doi.org/10.1002/(SICI)1521-3773(19990503)38:9 $<1172:$ :AID-ANIE1172>3.0.CO;2-C

12. F. Perret, A. N. Lazar, A. W. Coleman, Chem. Commun. 2006, 2425-2438. http://dx.doi.org/10.1039/b600720c

13. S. O. Cherenok, O. A. Yushchenko, V. Y. Tanchuk, I. M. Mischenko, N. V. Samus, O. V. Ruban, Y. I. Matvieiev, J. A. Karpenko, V. P. Kukhar, A. I. Vovk, V. I. Kalchenko, Arkivoc 2012, (iv), 278-298.

14. A. Casnati, F. Sansone, R. Ungaro, Acc. Chem. Res. 2003, 36, 246-254. http://dx.doi.org/10.1021/ar0200798
15. M. I. Husain, A. Kumar, R. C. Srivastava, Current. Sci. 1986, 55, 644-646.

16. S. H. Singh, L. D. S. Yadav, Agr. Biol. Chem. 1976, 40, $17-21$.

17. N. Ulusoy, Ö. Ateş, Ö. Küçükbasmacý, M. Kiraz, Y. Yeğenoğlu, Monatsh. Chem. 2003, 134, 465-474. http://dx.doi.org/10.1007/s00706-002-0546-z

19. V. A. Adhikari, V. V. Badiger, Ind. J. Chem. 1988, 27, 542- 547.

20. C. S. Andotra, B. S. Manhas, Acta Cienc. Indica Chem. 1992, 18, 99.

21. I. R. Ezabadi, C. Camoutsis, P. Zoumpoulakis, A. Geronikaki, M. Soković, J. Glamočilija, A. Ćirić, Bioorg. Med. Chem. 2008, 16, 1150-1161. http://dx.doi.org/10.1016/j.bmc.2007.10.082

22. N. G. Aher, V. S. Pore, N. N. Mishra, A. Kumar, P. K. Shukla, A. Sharma, M. K. Bhat, Med. Chem. Lett. 2009, 19, 759763. http://dx.doi.org/10.1016/j.bmcl.2008.12.026

23. A. A. El-Emam, O. A. Al-Deeb, M. Al-Omar, J. Lehmann, Bioorg. Med. Chem. 2004, 12, 5107-5113. http://dx.doi.org/10.1016/j.bmc.2004.07.033

24. S. L. Gaonkar, K. M. L. Rai, B. Prabhuswamy, Eur. J. Med. Chem. 2006, 41, 841-846. http://dx.doi.org/10.1016/j.ejmech.2006.03.002

25. B. Chandrakantha, P. Shetty, V. Nambiyar, N. A. M. Isloor, Eur. J. Med. Chem. 2010, 45, 1206-1210. http://dx.doi.org/10.1016/j.ejmech.2009.11.046

26. B. Silvestrini, C. Pagatti, Br. J. Pharmacol. 1961, 16, 209217.

27. D. Chauhan, J. S. Chauhan, J. Singh, S. K. Bajpai, M. N. Joshi, Indian J. Chem. 2003, 42B, 215-218.

28. S. Ilkay, S. Rollas, M. Kiraz, Med. Chem. Lett. 2001, 11, 1703-1707. http://dx.doi.org/10.1016/S0960-894X(01)00283-9

29. C. D. Gutsche, M. Iqbal, Org. Synth. 1990, 68, 234-236. http://dx.doi.org/10.15227/orgsyn.068.0234

30. S. Karakurt, T. F. Kellici, T. Mavromoustakos, A. G. Tzakos, M. Yilmaz, Curr. Org. Chem. 2016, 20, 1043-1057. http://dx.doi.org/10.2174/1385272820666151211192249

31. K. Akbari Dilmaghani, F. Nasuhi Pur, N. Hoseini Jazani, A. Alavi, Z. Niknam, F. Mirfakahraee, Phosphorus, Sulfur Silicon Relat. Elem. 2014, 189, 81-87. http://dx.doi.org/10.1080/10426507.2013.789877

32. K. Akbari Dilmaghani, F. Nasuhi Pur, M. Hatami Nezhad, Iran. J. Pharm. Res. 2015, 14, 693-699.

33. K. Akbari Dilmaghani, F. Nasuhi Pur, M. Mahammadpour, J. Mahammadnejad, Iran. J. Pharm. Res. In Press 2016.

34. (a) V. I. Cohen, J. Heterocycl. Chem. 1978, 15, 237-240. http://dx.doi.org/10.1002/jhet.5570150211

(b) K. Sung, A. R. Lee, J. Heterocycl. Chem. 1992, 29, 1101-1109. http://dx.doi.org/10.1002/jhet.5570290512

(c) R. Milcent, T. H. Nguyen, J. Heterocycl. Chem. 1986, 23, 881-883. http://dx.doi.org/10.1002/jhet.5570230343

35. R. Singh, A. Chouhan, World J. Pharm. and Pharmaceu. Sci. 2014, 3, 1474-1505.

36. J. Elquro, C. Marzin, A. R. Katritzky, P. Linda, Academic Press, London. 1976, 43, 33-36. 
37. R. Iqbal, K. H. Zamani, N. H. Rama, Turk. J. Chem. 1996, 20, 295-301.

38. (a) I. K. J. Al-Joubory, T. F. K. Albayati, K. A. J. Albayati, T. S. M. Al-Joubory, Diyala. J. Pure.Sci. 2013, 9, 47-56. (b) C. H. Lee, H. I. Cho, K. J. Lee, Bull. Korean Chem. Soc. 2001, 22, 1153-1155.

39. Z. T. Li, G. Z. Ji, C. X. Zhao, S. D. Yuan, H. Ding, C. Huang, A. L. Du, M. Wei, J. Org. Chem. 1999, 64, 3572-3584. http://dx.doi.org/10.1021/jo9824100

40. C. Guimon, G. Pfister-Guillouzo, Tetrahedron 1974, 30, 3831-3838.

http://dx.doi.org/10.1016/S0040-4020(01)97072-3

41. F. Malbec, R. Milcent, G. Barbier, J. Heterocycl. Chem. 1984, 21, 1689-1698.

http://dx.doi.org/10.1002/jhet.5570210624

42. V. I. Kelarev, G. A. Shvekhgeimer, A. F. Lunin, Khim. Geterotsikl. Soedin. 1984, 1271-1276.

43. S. Gopinathan, S. A. Pardhy, A. P. Budhkar, C. Gopinathan,
Synth. React. Inorg. Metal. Org. Chem. 1988, 18, 823-836. http://dx.doi.org/10.1080/00945718808060824

44. L. Mandolini, R. Ungaro, Calixarenes in Action, Imperial College Press, London, 2000.

http://dx.doi.org/10.1142/p168

45. M. Iqbal, T. Mangiafico, C. D. Gutsche, Tetrahedron 1987, 43, 4917-4930. http://dx.doi.org/10.1016/S0040-4020(01)87673-0

46. C. D. Gutsche, B. Dhawan, No, K. H. Levine, L. Bawer, Tetrahedron 1983, 39, 409-426.

http://dx.doi.org/10.1016/S0040-4020(01)88541-0

47. C. D. Gutsche, Acc. Chem. Res. 1983, 16, 161-170. http://dx.doi.org/10.1021/ar00089a003

48. C. Jaime, J. de Mendza, P. Prados, P. M. Nieto, C. Sanchez, J. Org. Chem. 1991, 56, 3372-3376. http://dx.doi.org/10.1021/jo00010a036

49. D. D Perrin, W. L. F. Armarego, Purification of Laboratory Chemicals, Pergamon Press, Oxford. 1988.

\section{Povzetek}

$\mathrm{Z}$ reakcijo 1,2,4-triazol-5-tiona oz. 1,3,4-oksadiazol-5-tiona s 5,11,17,23-tetra-terc-butil-25,27-bis(3-bromopropoksi)26,28-dihidroksikaliks[4]arenom (2) smo sintetizirali serijo tetra-terc-butilkaliks[4]arenov derivatiziranih z 1,2,4-triazol-5-tionskimi oz. 1,3,4-oksadiazol-5-tionskimi fragmenti na spodnjem robu kaliksarena. Pripravljene spojine smo karakterizirali z FT-IR, ${ }^{1} \mathrm{H}$ NMR, ${ }^{13} \mathrm{C}$ NMR spektroskopskimi podatki, elementno analizo in ESI-MS. 